\title{
Zeichnen als Weltentwurf: Analog + Digital - Die Bedeutung des Zeichnens in der Primarausbildung mit Blick auf Design Engineering in Europa
}

Marina-Elena Wachs

"Sketching the world" bedeutet entwerfen von Welt mittels analogen und digitalen Zeichenmitteln - auch in Zukunft benötigt es dafür im Design Engineering der Künstlichen Intelligenz (KI) und der Emotionalen Intelligenz (EQ - nach Daniel Goleman). Zeichnen ist Kulturgut seit der Steinzeit und ist für die kulturelle Bildung als auch für das Design Engineering der Zukunft von großer Bedeutung. Zeichnen-Erfahrungen, sei es mit dem Stock im Sand oder mit dem Stift auf Papier, beginnt in der Primarbildung: Bereits hier ist es als Kulturtechnik essentiell für die Gesellschaft, indem der aktive Akt des Zeichnens Selbstausdruck und kulturelles Gedächtnis, Signifikat ist. Zeichnen ist individuelles und kollektives Wissens- und Bildungsmanagement für die Welt von morgen und muss in der Primabildung früh - multidisziplinär - verankert sein, soll es im Skizzieren, im Mapping, als Repräsentation und Projektion wirkungsvoll für das Design Engineering in Europa, auch in der Augmented Reality, eingesetzt werden.

Keywords: Zeichnen als Wissensarchiv, Wissensmanagement versus Datenmanagement; analoges + digitales Zeichnen in der Primarausbildung; Curriculare Schulbildung im 21. Jh. benötigt Design Engineering-Fächer; mapping the world in sketching - ein Revalue der Kulturtechniken

\section{Einleitung}

Blicken wir auf die ersten Erfahrungen des Zeichnens, mit der Hand in den Sand, zum Beispiel während der Kleinkindphase (vgl.: Elschenbroich, D. 2001), müssen wir erkennen, dass wir uns ein Bild machen von der Welt und zugleich ein Abbild schaffen von dem, was um uns real ist, aber auch in Koexistenz mit den Gefühlen, assoziativen Energien und Bildern in uns als Individuum. Aus neurowissenschaftlicher Perspektive (vgl.: Hüther, G, 2009, S. 81 ff) wird deutlich, dass die vernetzten Areale von Hand und Gehirn 
(vgl. Wehr, M. \& Weinmann, M., Hrsg., 2005), die Synapsen-Bildung während der Kleinkindphase und ein Lernen mit allen Sinnen, die Voraussetzung für das Designen der Zukunft ist. Es ermöglicht uns, Bilder nicht allein zu exponieren, sondern komplexe Muster zu interpretieren. Es ermöglicht ein Abstraktionsvermögen für den Erwachsenen zu bilden, das signifikant für das Ingenieurswesen und Wissensmanagement der Gesellschaft ist.

\section{Analog und digital Zeichnen - Die Bedeutung der Hand/-Zeichnung in der Primarbildung, für das Ego und für das Ingenieurswesen}

Das Phänomen des „thinking through drawing“ (vgl.: Kantrowitz, A., 2016), erlangte seit der Jahrtausendwende ebenso als Designmethode größere Bedeutung. Es ist bedeutsam für die Kompetenz eines complex problem solving, das im Zuge der voranschreitenden Digitalisierung, als eines der Phänomene bezeichnet wird, das den größten Kompetenzbereich des Designers und Ingenieurs der Zukunft ausmacht (vgl. Schwab, K. 2016, ebenso Günthner, R., Dollinger, D., 2019). Vom Beginn der Steinzeit und der Höhlenmalerei, über die Renaissancezeit, in der Leonardo Da Vinci bedeutende Flugobjekte zeichnend konstruierte, bis hin zum computer added design und virtuellen Skizzieren heute mittels controller, bedarf es eines Vorstellungsvermögens, bei dem die Skizze das Medium der Vermittlung ist. Die Skizze ist Grundlage der Diskussion, ein Kommunikationsmittel.

Designen meint Selbstexpression und Vermittlung der Idee, die zunächst nur im Kopf des Individuums ist und Produkt- oder Prozess-Darstellung werden muss. Dieser Prozess beginnt mit dem Reproduzieren von weltlichen Dingen um uns herum und von inneren Bildern des Menschen. Hand und Hirn (vgl. Günthner, R., Dollinger, D., 2019, S. 101) gehen Hand in Hand (Wehr, M., Weinmann, M., 2009, S. 16 f), um Produktsprache und linguistische Sprache zu formen (Wachs, M.-E., 2008, S. 2017). "Jedes normale Kind macht im Laufe seiner Entwicklung die Erfahrung, dass man Repräsentationen von Dingen oder Vorgängen in der Außenwelt Zeichen, gesprochene oder geschriebene Wörter, zuordnen kann und dass diese Zeichen es ermöglichen, mehr über die Welt zu erfahren, sie zu kategorisieren. Konzepte von dieser Welt zu entwickeln [...] im Zuge des Spracherwerbs, der taktilen, visuellen und akustischen Auseinandersetzung mit der Welt [...]." (Weinmann, M., in: Wehr, M., Weinmann, M., 2009, S. 16) ermöglichen Erkenntnis über dieselbe und einen multidisziplinären Spracherwerb. Es werden somit Zeichen- und Sprachsysteme identifizierbar, über das erste Abgreifen mit der Hand, über die Identifizierung von Texturen und Tönen, die assoziativ mit anderen Systemen abgeglichen und sowohl erinnert werden als auch als Repertoire zur Generierung neuer (Produkt-) Sprachen und 
Konzepte genutzt werden. Insofern sind Zeich(n)en-tools Werkzeuge für das Kreieren neuer Lösungen und zugleich Selbstausdruck und Selbstbewusstwerdung des Individuums, das für die "industrielle“ Lösungsskizzierung essentiell ist. Hierin kommt der Konzeptdarstellung durch Mapping eine besondere Bedeutung bei, auf die in Kapitel vier vertieft eingegangen wird.

\section{Strategien der Design-Ausbildung}

\section{Industrial-Design-Ausbildung der Vergangenheit}

Blicken wir auf die Geschichte der Industrie-Design Ausbildung der deutschen und nordischen Länder Europas wird deutlich, dass wir in Deutschland aus dem Erbe der Werkkunstschulen profitieren, die ebenso vernetzt waren mit Handwerksbetrieben, handwerklichen Ausbildungsstrukturen der universitären Bildung - mittels Praktika in Werkstätten bspw. zu den Werkstoffen Holz, Kunststoff, Metall oder Glas - und in Beziehung zu industriellen Auftragsarbeiten und Kooperationen standen. Ein ähnliches Bild, bzw. eine ähnliche Identifikation des Industrial Design-Standes und -Ausbildung geht ebenso in Groß Britannien aus der Nachkriegszeit hervor, durch eine europäische Befruchtung dieser Kompetenzen, mit ehemaligen Designern des Bauhauses. Somit lässt sich bis weit in die 1990er Jahre hinein ein ähnliches Bild der Industrial-Design Ausbildung in den nordischen Ländern Europas und ein ähnliches, gemeinsames Verständnis von Design Engineering konstatieren. (vgl.: Wachs, M.-E., Hall, A., 2019)

Ein Verständnis oder eine Verständigung der Lesbarkeit von codes, von Produktsprachen ist Basis für das Lesen von Designsprachen und komplexen Systemen der Zukunft. Seit den 1990er Jahren lässt sich eine Tendenz zu mehr designtheoretischen Designmethoden zur Generierung von Design-Lösungen ausmachen. Beispielsweise nutzen Methoden wie "thinking design“ nach Rittel und "systems oriented design" nach Maedows, kombinative Zeichenmethoden wie mind mapping und sprachliche Vernetzung, die zum späteren Zeitpunkt in Produkte verwandelt werden. Dieses Verhalten hat in viele Branchen Einzug gehalten, indem mittels einer Art Piktogramm-Zeichensprache kommuniziert wird, zudem die Skills dieser eher Comic-haften Zeichensprache ein jede/r trainieren kann (vgl. Raom, D., 2009).

Es hat sich gezeigt, dass die Design- und Ingenieurs-Ausbildungen sich parallel zu den industriellen und gesellschaftlichen Veränderungen entwickeln. Mit zunehmendem Druck in der Forderung nach nachhaltigen Systemen ist ein entrepreneurial university -Bestreben sichtbar, das auch mittels der Digitalisierungsphase interlinked learning landscapes kreiert, die zum einen allen Menschen eine Teilhabe an Wissen ermöglichen 
aber ebenso am Wandel des cultural behaviour, das Produkte und Konzepte auf Nachhaltigkeit hin stärker wieder untersucht. Diese interaktiven Lernlandschaften sind von Künstlicher Intelligenz (KI) geprägt. Eine KI im Bereich des Design Engineering kann jedoch nur so gut gespeist, geführt werden und agieren, wie die Menschen diese Systeme generieren auch entsprechende Kompetenzen erlangen, zu dem auch eine ethische Frage gehört, die die Emotionale Intelligenz (EQ) integriert. Insofern ist ein Zeichnen von neuen Designlösungen auch innerhalb dieser digitalen Ebenen immer ein Resultat des Einzelnen und seiner praktischen (technischen) als auch epistemologischen Erfahrungen bezüglich der Gesellschaft.

\section{Design Ingenieur Bildung der Zukunft mit Blick auf KI und EQ - Mit textilem Material skizzieren - Materialising immateriality}

Eine Zunahme der designtheoretischen Designmethoden geht einher mit: 1. einer Immaterialisierung von Welt, indem wir Dinge nur noch leasen oder mit Hilfe digitaler Programme und Avataren weltliche Dinge simulieren, um Ressourcen einzusparen; 2. einem Wandel des soziologisch bedeutsamen material and cultural behaviour (vgl.: Wachs, M.-E., 2008, S. 268). Der Online Konsum und weitere mediale Produktnutzung untermauern den „Design Shift" (Wachs, M.-E., 2018, S. 398), mit denen wichtige Kompetenzen verloren gehen.

Die folgende Abbildung zeigt das Ergebnis eines Workshops, dass mit Hilfe von textilen Materialien unterschiedliche Zustände, Verläufe und Integration von Prozessparametern - und -Teilnehmern der Krankheit Demenz ausdrückt. Es handelt sich um ein Skizzieren mit Hilfe textiler Materialien - einem design doing durch "materialising immateriality" (Wachs, M.-E., 2019), indem das theoretische Wissen über die Krankheit und deren Verlauf mittels differenzierter haptischer Oberflächen, Texturen, Materialqualitäten und -Farben ausgedrückt wird.

Das Ziel ist es, mittels materialbasierten Skizzierens die Ideen, Konzepte auszudrücken, zu kommunizieren, zu visualisieren, zu vermitteln, als ebenso die in den Designprozess beteiligten Menschen über eine Diskussion zu involvieren, und zugleich die eigene Idee (im Prozess) zu evaluieren: Im Skizzieren - mittels Material - ein Anschauungsmedium zu schaffen, dass die Designthematik reflektiert und zu einer guten Lösung führt.

Diese Fallstudie wurde deshalb gewählt, da die Doktorarbeit im Design aufzeigt, die Krankheit Demenz einer Designlösung zuzuführen, die ebenso System-Verständnis als auch Empathie-Verständnis benötigt und da sie die Ebene der Emotionen relevant einbindet. Darüber hinaus ist es aber auch die emotionale Intelligenz des Menschen allgemein, die hier in die "Zeichentechnik" eingreift. Es ist eine integrative Aufgabe, die 
verschiedene Intelligenzebenen bei der Lösungsfindung berücksichtigen muss, wenn am Ende auch die Künstliche Intelligenz für ein Service-Design des autark lebenden, dementen Menschen die Medikamentengabe und -Verwaltung integrieren sollte. Es ist nur ein Beispiel, wie das Skizzieren von Ideen, von immateriellen Vorstellungen, mittels Material und digitalen Medien in Zukunft interaktiv analoge und digitale Systeme nachhaltig miteinander verknüpfen muss und verdeutlicht, dass Hand und Hirn aber auch die folgenden Intelligenzebenen zusammenspielen (Abb. 2, 3).

\section{Zeichnen als Wissensarchiv - Zeichnen mit der Hand: bedeutsam für Industrie und Wissensmanagement der Zukunft}

\section{Welt einordnen, strukturieren, kategorisieren mittels Zeich(n)en + der didaktische Mehrwert im Design}

„Das Wissen um die Artefakte lässt die Menschen ihre Kultur erkennen und einordnen, die Materialität der Objekte offenbart ihre Bedeutung, deren wir uns [Anm.: laut Elsbeth Stern, 2001] assoziativ erinnern. Dies ist kein abstrakter Vorgang, sondern auf die Handlungsweisen im Umgang mit den Dingen zurückzuführen, wenngleich sich die "Ordnung der Dinge" nach Michel Foucault ebenso sprachlich determinieren lässt." (Wachs, M.-E., 2008, S. 204) Die Welt, die Dinge, die wir zeichnend reproduzieren, abgreifen um zu begreifen, stellen unser kulturelles Gedächtnis dar, und im Akt des Zeichnens repräsentieren sich Kulturtechnik und Selbstexpression zugleich. Zeichnen beschreibt einen kulturellen Akt des Strukturierens und Dokumentierens, Skizzieren von Welt - und eines Managements des Wissens und der Bildung für die Welt von morgen - auch innerhalb der Ingenieurswissenschaften. Das bedeutet, dass wir nicht allein die MINT -Fächer in den Fokus nehmen sollten, sondern ebenso die Techniken der Künste wieder stärker in das Blickfeld einer nachhaltigen Bildungsstrategie einbeziehen. Es bedeutet erneut die analogen, manuellen Kenntnisse in der digitalen Welt der "digital natives" (vgl. Abb. 3 „digital native“) zu befördern, um das BeGREIFEN von morgen zu ermöglichen.

Der in diesem Text eindringliche Appell, zu einer erneuten manuellen Befähigung der Kinder und Jugendlichen zur Selbstexpression durch Zeichnen mit der Hand, dient nicht allein dem didaktischen Mehrwert in der bildnerisch-ästhetischen Praxis im Kunstunterricht. Es dient vor allem der Rezeptions-Befähigung, der Bildung von Abstraktionsvermögen und dem Lesen komplexer Muster und Systeme des einzelnen und folglich einem didaktischen Mehrwert im Design(en). „Der Beginn der menschlichen Frühformen des Zeichnens liegt im Zusammentreffen einer Bewegungsdynamik mit einer 
Oberfläche, die diese Dynamik festhält" (vgl.: Peez, G., 2018, S. 119). Diese aktive Zeichenform ist genauso relevant für das Zeichnen mit dem Controller in der Augmented Reality (vgl. Seymour-Powell, 2017, online), wie es analog in der schnell dargestellten 2D Zeichenebene der (digitale) Duktus sein kann. Ein Nachzeichnen der realen Welt ist wesentliche Voraussetzung für eine Ideenfindung und -generierung von neuer Welt im virtuellen Raum - human centred designing. Die Didaktik des Zeichnens während der Zeit der Kindheitsprägung kann in den Bildungswissenschaften nicht groß genug geschätzt werden, für die Bedeutung als Vorbildung für den weiterführenden DesignUnterricht in den Schulen und Universitäten und letztendlich für das Ingenieurswesen in Europa.

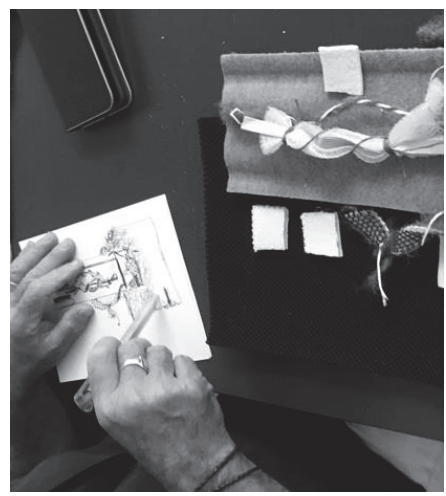

Abbildung 1: Veranschaulichung von Designaufgaben und -ideen durch "Materialising Immateriality" 2019, M.-E. Wachs

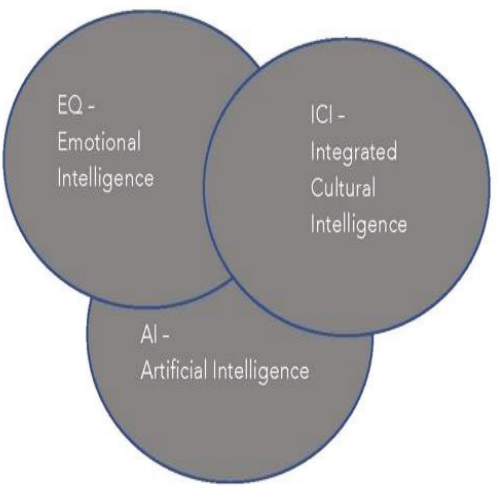

Abbildung 2: $E Q-I C l-A l$-Zusammenspiel im Designprozess, 2021, M.-E. Wachs.

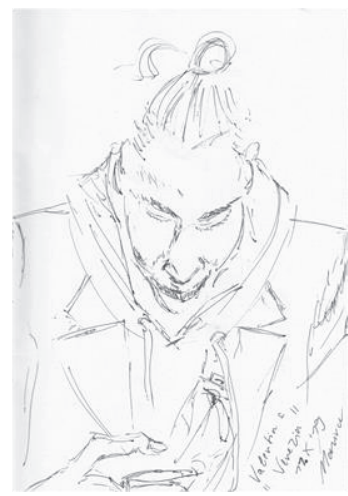

Abbildung 3: "digital native", Skizze verdeutlicht rudimentäre Kompetenz der "Hand (Daumen)-Hirn -Funktion", 2021, M.-E. Wachs.

\section{Revalue Mapping als Kulturtechnik von morgen - oder wieviel Kunst be- nötigt das Design Engineering und wieviel Design benötigt die Kunst?}

Kommen wir zurück zum Beginn der Ausführungen: sketching the world ist essentiell für ein Mapping the world in sketching, und hierbei ist "in“ eine Anlehnung an lean inoder Skin in the game Management Strategien. Die Kompetenz des Zeichnens kann ein Strategie tool sein, als Strategie zum Begreifen von Welt, zum Strukturieren von Wissen, zur Kategorisierung und Archivieren von Wissen und wird täglich im Design in der Technik des brainstorming, mood boarding, design thinking oder im mind mapping an sich 
angewandt. Mapping aus Sicht der Kunstpädagogik ist essentiell für die Design- und Ingenieurswelten der Zukunft.

Es bedarf einer Wiederbelegung des Skizzierens und dem revalue des Mapping als Kulturtechniken im Kunstunterricht, um sich seiner selbst und den Wissensarchiven von Unternehmen, Prozessen und Gesellschaft zu vergegenwärtigen; und das benötigt auch in Zukunft das Können und Training der Hand, die abgreift, nachformt, simuliert, reflektiert, um neue Ideen zu generieren und zu kommunizieren. „Mapping und Kartierung werden als Methoden der Erkenntnisgewinnung, Gestaltung und Präsentation sowohl in der Kunst als auch in den Wissenschaften angewandt. Zugleich ist Mapping im weitesten Sinne ein Verfahren [...]", das für Strukturbilder im Alltag und "[...] als kreative Technik in der Pädagogik (Brainstorming [...] eine Rolle spielt. Der zentrale Punkt des Mappings ist die Visualisierung von assoziativen Bezügen zwischen unterschiedlichen Phänomenen [...]. Das Ergebnis eines solchen Mapping-Prozesses nennt man u.a. >Atlas<." (Peetz, G, 2018, S. 112).

Mapping aus Sicht der Wissenschaftsphilosophie beschreibt den didaktischen Mehrwert für den Konstruierenden - Designer - und den User - Leser - der Karte (map): „A map organizes the entire narrative, both for the author in the process of composition and for the reader in the process of comprehension." (Grønfeldt Winther, R., 2020, S.2). Derartig skizzierte Karten, maps sind kulturelle Werkzeuge und Zeugnis zugleich, sie sind Projektions- und Repräsentationsmittel - Repräsentationen unserer Selbst und des Weltzustands: Mapping the world by sketching!

„Our representations [...] influence our desire and our angst, and our imagination, [...] shaping the possible futures of our children [...] scientific representations will inform the strategies we as society design [...]" (Grønfeldt Winther, R., 2020, S.243f). Die ästhetische und somit funktionale Qualität und somit Lesbarkeit von Repräsentationen wie beispielsweise Karten - sind abhängig von der Design-Qualität (und Kompetenzen der Desing-ExpertInnen) wie das Design(en) der Zukunft von der künstlerischen und kulturellen (Vor-)Bildung abhängig ist, um jeglichen Ausdruck von (Produkt-) Sprachen nachhaltig und gesellschaftsrelevant zu skizzieren, zu entwerfen.

\section{Literaturverzeichnis}

Elschenbroich, D. (2001): Weltwissen der Siebenjährigen - Wie Kinder die Welt entdecken können, Goldmann Verlag.

Grønfeldt Winther, R., (2020), When Maps Become The World, Chicago: The University of Chicago Press.

Günthner R., Dollinger, D. (2019), Hirn 1.0 trifft Technologie 4.0 - Der Mensch und seine kreativen Potentiale im Fokus,, Springer Verlag 
Hüther, G., 2009 (2004), Die Macht der inneren Bilder - Wie Visionen das Gehirn, den Menschen und die Welt verändern, Vandenhoeck \& Ruprecht.

Kantrowitz, A. (2016): We all draw: The fourth international thinking through Drawing Symposium“, Drawing: Research, Theory, Practice, vol. 1, no. 2, 2016, p. 282.

Maedows, D. H., (2008) Wright, Diana (Ed.), 2009, Thinking in Systems, A Primer, Earthscan.

Peez, G., (2018), Einführung in die Kunstpädagogik, Stuttgart: Kohlhammer.

Roam, D., (2009), The Back of the Napkin - Solving Problems and Selling Ideas with Pictures, Penguin.

Schwab, K., (2016), The Fourth Industrial Revolution, Geneva: Penguins, p. 42.

Seymour Powell, (2017), Reality works: https://www.youtube.com/watch?v=j5bAig-8uFs, abgerufen am 06.10.2020

Wachs, M.-E., (2008), Material Mind - Materialgedächtnis, Neue Materialien in Design, Kunst und Architektur, Hamburg: Dr. Kovaç Verlag.

Wachs, M.-E., (2018), Driver for sustainable (industrial) design culture - the >design shift<, EPDE Congress, London, p. 394-399.

Wachs, M.E., Hall, A., (2019), European Driving Range - innovative landscapes for a tangible, non-hierarchical learning space within a material and immaterial togetherness, EPDE Conference, Glasgow, Scotland.

Wachs, M.-E., (2019), Keynote Introduction for interdisciplinary design engineering workshop: at Royal College of Art, London, School of Design. - Anmerkung: die Ergebnisse werden in post doc thesis erscheinen: Wachs, M.-E. (2021) Design Engineering - Yesterdays Future, Niggli Verlag.

Wachs, M.-E., (2021), Design Engineering - Yesterdays Future, Schweiz: Niggli Verlag.

Wehr, M. und Weinmann, M. (Hrsg.) (2005), Die Hand - Werkzeug des Geistes, Spinger.

Wilkinson, C., (2015), RA on thinking through drawing, copyright Candida Richardson, https://www.royalacademy.org.uk/exhibition/chris-wilkinson-thinking-through-drawing, abgerufen am 06.10.2020.

\section{Kontakt}

Prof. Dr. phil. Dipl.-Des. Marina-Elena Wachs

Hochschule Niederrhein

Webschulstr. 31

41065 Mönchengladbach

marina.wachs@hs-niederrhein.de 